\title{
Transnationale Lernprozesse - Erfahrungen mit der grenzüberschreitenden Verbreitung gewerkschaftlicher Strategien
}

Haben die Gewerkschaftstage von ver.di und der IG Metall Aufbruchsstimmung verbreitet? Die Antwort hieße Nein, wenn man deutliche Umwidmungen von finanziellen und personellen Ressourcen als Maßstab für eine grundlegende strategische Neuorientierung setzt. Dennoch finden in beiden Gewerkschaften strategische Erneuerungen statt, die überdies in unterschiedlicher Weise Erfahrungen von Gewerkschaften außerhalb Deutschlands aufgreifen. In diesem Zusammenhang werden derzeit die amerikanischen Konzepte des Organizing und der „strategischen Kampagne“ diskutiert. Was verbirgt sich hinter diesen Konzepten und welche Erfahrungen wurden bisher in Deutschland mit ihnen gemacht? ${ }^{1}$

\section{Wie lernen Organisationen?}

Strategische Neuausrichtungen haben stets mit Lernprozessen zu tun. Jedoch: Gewerkschaften werden von der diesbezüglichen organisationssoziologischen Debatte nur selten als „lernende Organisationen“ (Zoll 2003) erfasst; im Vordergrund stehen staatliche Organisationen und Unternehmen. Es gibt in der Tat wichtige Besonderheiten von Gewerkschaften, welche die Anwendung von Erkenntnissen aus der Analyse anderer Organisationen erschweren, insbesondere die innerorganisatorischen demokratischen Strukturen. Dennoch finden sich in der Debatte, für deren breite Diskussion hier kein Platz ist (für einen allgemeinen Überblick zum Organisationslernen vgl. Bandelow 2003; für Gewerkschaften vgl. Hyman 2007), einige zentrale Einsichten:

Erstens lernen Organisationen vor allem über diejenigen Individuen, die innerhalb der Organisation Wissen etablieren und verbreiten können. Zweitens dienen die in den Handlungsanleitungen der Organisation verankerten Grundsätze als institutionelles Gedächtnis, das über den Einfluss der Individuen hinaus dem erlernten Wissen Geltung verschafft. Drittens können verschiedene Lerntypen unterschieden werden. Beim Lernen vom Typ 1 verbleibt die Organisation innerhalb ihres bestehenden strategischen Paradigmas; es geht um das Erlernen neuer Techniken zur Verfolgung etablierter Ziele. Voraussetzung für Lernen vom Typ 2 ist eine Veränderung des strategischen Paradigmas und damit auch der Ziele der Organisation. Typ 3 schließlich bezeichnet die Etablierung von Strukturen, die der Organisation gewissermaßen erlauben, das Lernen selbst zu lernen. ${ }^{2}$

Viertens ist Lernen immer auch ein politischer Prozess. Apriori bestehende innerorganisatorische politische Interessen und Widerstände bestimmen darüber, welche Strategien adaptiert werden, auf welche Weise sie zur Anwendung kommen und wie dies diskursiv präsentiert wird. Dabei können unbequeme Anregungen, die ein Lernen vom Typ 2 nahelegen, oft nur von außerhalb der Organisation kommen, entweder auf der Basis von (scheinbar) überlegener Expertise (Stichwort Unternehmensberatungen) oder von erfolgreichen Erfahrungsbeispielen. Vor allem Letztere können auch von außerhalb des eigenen nationalen Kontexts stammen, wobei dann von den organisationsinternen Promotoren oft unterschlagen wird, dass die Erfolge institutionell und kulturell voraussetzungsvoll sind. Diese Form von diskursiver "Rosinenpickerei“ ist in Deutschland u.a. bei der Diskussion um den Transfer angeblich überlegener Arbeitsmarktpolitik bekannt (Scherrer 2005).

Für die Analyse innerorganisatorischer Lernprozesse bietet sich neben organisationssoziologischen Ansätzen wie dem Modell der "Advocacy Coalitions“ (Sabatier 1993) oder dem des „Lesson-drawing“ (Rose 1991) auch Antonio Gramscis Konzept des „organischen Intellektuellen“ an, welches die Zentralität von Führungspersönlichkeiten innerhalb der Organisation betont (für den Versuch einer Operationalisierung vgl. Greven 2003). Eine ausführliche Analyse der Lernprozesse in deutschen Gewerkschaften bezüglich strategischer Erneuerung und insbesondere der transnationalen Elemente solcher Prozesse kann hier nicht geleistet werden. In der Folge soll aber versucht werden, auf der Basis von Dokumentenanalysen und teilnehmender Beobachtung erste Einsichten aus jüngeren Erfahrungen mit dem Transfer US-amerikanischer Strategieelemente zu formulieren. Zunächst ist zu klären, warum der

\footnotetext{
Dieser Beitrag enthält erste Ergebnisse eines von der Hans-Böckler-Stiftung geförderten Projekts zur Transnationalisierung des gewerkschaftlichen Organisationslernens.

2 In der Literatur gibt es für die drei Typen zahlreiche verschiedene Bezeichnungen (z. B. Einschleifen und Doppelschleifen-Lernen bei Argyris/ Schön 1999) mit Abweichungen bei der Definition, die hier aber außer Acht bleiben sollen.
}

Thomas Greven, Dr., Politikwissenschaftler Senior Research Fellow am Institut für Internationale Politik, Berlin, Lehrbeauftragter am John F. Kennedy-Institut für Nordamerikastudien der FU Berlin und Mitglied von Organisierung \& Kampagnen (OrKa). Arbeitsschwerpunkte: Gewerkschaftspolitik in der Globalisierung, amerikanische Innenpolitik.

e-mail: tgreven@zedat.fu-berlin.de Wilfried Schwetz, Diplom-Sozialwirt, Labour Policies and Globalisation (M. A.). Arbeitsschwerpunkte: Organizing und strategische Kampagnen.

e-mail: Wilfried.Schwetz@htp-tel.de 
Strategietransfer ausgerechnet aus den USA erfolgt und welche besonderen Bedingungen für grenzüberschreitendes Lernen gelten.

\section{Lernen von den USA - warum?}

In den letzten beiden Dekaden ist eine breite Debatte über die Ursachen schwindenden Einflusses der Arbeiterbewegung in Staat und Wirtschaft aufgekommen. Strukturell orientierte Ansätze sehen die Ursachen in sozialen und ökonomischen Veränderungen, Arbeitsmarktreformen und wachsender Gewerkschaftsfeindlichkeit. Diese Ansätze tendieren jedoch dazu, Gewerkschaften als „passive Empfänger von Veränderungen in ihrer Umwelt" (Visser 1994, S. 84) anstatt als „Architekten ihres eigenen Schicksals“ (Frege 1999, Übersetzung nach Dribbusch 2003, S. 16) zu betrachten. Dagegen machen stärker akteursorientierte Ansätze nicht allein äußere Faktoren für den schwindenden Einfluss verantwortlich, sondern sehen diesen auch im Handeln der Arbeitnehmerorganisationen selbst begründet. ${ }^{3}$ Letztere Ansätze sind eng verbunden mit der internationalen, vorwiegend angelsächsischen Debatte über Gewerkschaftsrevitalisierung (Bronfenbrenner et al. 1998; Fairbrother/Yates 2003; Turner et al. 2001; Hurd et al. 2003; Milkman/Voss 2004), in der der Begriff „organizing" eine zentrale Rolle spielt.

Im angloamerikanischen Sprachgebrauch meint "Organizing“ im engeren Sinne zunächst schlicht Mitgliederwerbung, die in den USA allerdings aufgrund der Regelungen zur betriebsbezogenen Anerkennung immer kollektive Mitgliedergewinnung ist. Weil der Organisationsgrad seit Jahrzehnten kontinuierlich sinkt, unter anderem da viele Unternehmen die gewerkschaftliche Organisierung massiv bekämpfen, gibt es seit Längerem eine Reformdebatte, die Gewerkschaften unter Druck setzt, die ihre Hauptaufgabe in Dienstleistungen für ihre Mitglieder sehen („Service Model“). Die Kritiker argumentieren, dass die effektive Erbringung dieser Dienstleistungen (vor allem guter Tarifverträge) auf Dauer nur gewährleistet werden kann, wenn die Gewerkschaften wieder über mehr Mitglieder verfügen. Daher müsse in verstärktes Organizing investiert werden.
In einem erweiterten Sinn meint Organizing sehr viel mehr als nur Mitgliederwerbung: Es geht um Aufbau und Aktivierung einer handlungs- und durchsetzungsfähigen gewerkschaftlichen Basis innerhalb und außerhalb der Betriebe. Die mobilisierten Beschäftigten erfahren sich - zumeist im Verlauf konkreter Konflikte, die aus ihrer Sicht wichtig sind - selbst als Gewerkschaft („ownership“). Sie werden so idealtypisch, gemeinsam mit den Hauptamtlichen, zu „Problemlösern“ von Konflikten, die sich darüber hinaus aktiv in der Mitgliederwerbung und in der politischen Arbeit engagieren. In diesem erweiterten Verständnis von Organizing verringert sich auch der Kontrast zwischen Organisierung und "Service“, weil insgesamt die Stellvertretungslogik aufgegeben wird (Banks/ Metzgar 1993).

Erstaunlich ist, dass auch außerhalb des angelsächsischen Raumes insbesondere das Organizing-Modell der US-Gewerkschaften aufgegriffen wird, obwohl diese mit insgesamt $12 \%$ und nur $8 \%$ in der Privatwirtschaft einen der schwächsten Organisationsgrade aller OECD-Länder aufweisen. Behrens (2005a) erklärt dies damit, dass auch Gewerkschaften, deren Macht weniger auf der Mobilisierung ihrer Mitgliederstärke als auf ihrer Institutionalisierung im politischen System basiert, Interesse an mitgliederorientierten Strategien entwickeln, wenn diese Institutionalisierung erodiert. Die US-Gewerkschaften mussten eher als andere auf Globalisierung und politische Angriffe reagieren, und sie mussten dafür ihre Mitglieder mobilisieren und neue gewinnen, weil ihre rechtliche und gesellschaftliche Verankerung stets prekär geblieben ist. Strategisch ergibt sich für Gewerkschaften, die ihre Macht hauptsächlich aus ihrer institutionellen Einbindung und günstigen rechtlichen Regelungen beziehen, die Herausforderung, auf die gegenwärtige Erosion ihrer rechtlichen und/oder institutionellen Machtquellen (in Deutschland unter anderem die Verhinderung des Aufbaus von betrieblicher Mitbestimmung, Austritte von Unternehmen aus Arbeitgeberverbänden oder Rückzug aus der Tarifbindung) adäquate Antworten zu finden. Rechtliche Rahmenbedingungen sind nur schwer zu verändern; Organisierungs-, Mobilisierungs- und Kampagnenfähigkeit sind dagegen Faktoren, die von Gewerkschaften unmittelbar beeinflusst werden können - wenn auch stets im Kontext der bestehenden rechtli- chen und institutionellen Bedingungen. Das Spektrum der Innovationen ist allerdings zunächst durch die oben beschriebene institutionelle Einbindung begrenzt (Behrens 2005b), wodurch Erfolgsbeispiele aus anderen systemischen Zusammenhängen eine besondere Bedeutung für den Innovationsprozess bekommen, können sie doch einen Sprung von Lernen des Typs 1 zum Typ 2 ermöglichen, d.h. die grundlegenden strategischen Paradigmen und Organisationsziele verändern.

Für Gewerkschaften ist das grenzüberschreitende Lernen bisher weniger selbstverständlich als für Unternehmen; sie sind trotz des traditionellen Bekenntnisses zum Internationalismus und ausgebauter internationaler Organisationen weiterhin Gefangene ihrer nationalen Bindungen und zudem durch die verschärfte Standortkonkurrenz in ihren transnationalen Aktivitäten behindert (Greven/Scherrer 2005). Auch in der Forschung wird die Perspektive des grenzüberschreitenden Lernens und der Adaption strategischer Innovationen von Gewerkschaften in anderen Ländern meist ausgeblendet - selbst bei Studien, die jenseits von strukturellen Faktoren auch „framing processes“ für die Auswahl von Erneuerungsstrategien verantwortlich machen (Frege/Kelly 2003).

Grundsätzlich treffen die lerntheoretischen Einsichten der Organisationssoziologie auch auf das grenzüberschreitende Lernen zu. Hinzu kommt eine Verstärkung von Problemen der institutionellen und kulturellen Passung. In der Debatte über Institutionentransfer ( $\mathrm{z} . \mathrm{Zt}$. vielfach unter dem Stichwort „Modell USA“) wird zu Recht darauf hingewiesen, dass die Übernahme einzelner Elemente bzw. Praktiken den historisch gewachsenen Zusammenhalt existierender Institutionen gefährden kann, wenn das Problem unterschiedlicher institutioneller und kultureller Kontexte zu wenig Beachtung findet (vgl. Scherrer 2005). Unbedacht übernommene Strategien können für Irritationen sorgen, wodurch traditionelle Strategien gegebenenfalls an Effektivität verlieren und existierende institutionelle und kulturelle Arrangements weiter erodieren. Die Innovationen können z. B. aufgrund der Unerfahrenheit der Akteure nicht die gewünschte Wirkung entfalten oder die erhofften positiven Wirkungen

\footnotetext{
3 Zum hier grundlegenden Konzept der "strategic
} choice" vgl. Kochan et al. 1986. 
etablierter Institutionen (wie z. B. Betriebsräte, Arbeitnehmervertretungen in Aufsichtsräten) konterkarieren. Beim Transfer von Strategien muss also die institutionelle und kulturelle Kompatibilität beachtet werden: Die Berücksichtigung der mit dem Problem der institutionellen und kulturellen Passung (,institutional fit“, ebd.) verbundenen Herausforderungen ist eine wesentliche Voraussetzung für erfolgreiche Transfers. Hierbei ist es vor allem wichtig, $\mathrm{zu}$ analysieren, wie im akademischen und innergewerkschaftlichen Diskurs mit dem Problem unterschiedlicher institutioneller und kultureller Kontexte umgegangen wird. Welche Beachtung wird den spezifischen Entstehungs- und Erfolgsbedingungen der zu importierenden Strategien geschenkt? Inwieweit wurden die Erfahrungen im Herkunftsland der Strategieinnovationen systematisch ausgewertet? In der gewerkschaftlichen Kampagnen-Praxis haben eben diese institutionellen und kulturellen Differenzen zu Problemen geführt. ${ }^{4}$

\section{Die amerikanische Diskussion um Kampagnen und Organizing}

Um zeigen zu können, inwiefern die institutionellen und kulturellen Voraussetzungen der Strategieinnovationen amerikanischer Gewerkschaften beim Transfer beachtet wurden, werden diese kurz im Überblick diskutiert. Die Debatte um die Erneuerung der US-Gewerkschaften begann in den späten 1970er und frühen 1980er Jahren nicht mit einer Diskussion um Organizing, sondern mit der umstrittenen Einführung von zunächst „corporate campaigns" genannten Kampagnenstrategien. Diese wurden von einigen US-Gewerkschaften entwickelt, weil aufgrund der prekären rechtlichen Situation und zunehmender unternehmerischer Angriffe traditionelle Arbeitskampfmaßnahmen wie Streiks immer weniger wirksam waren. In strategischen Kampagnen, so werden sie heute meist bezeichnet, wird die Tatsache genutzt, dass ein Unternehmen in eine Vielzahl von Beziehungen mit seiner Umwelt eingebunden ist (Kunden, Banken, Staat, Gewerkschaften, Zulieferer etc.) und alle diese Beziehungen Ansatzpunkte bieten, um gezielt und eskalierend Druck auf das Unternehmen aufzubauen (Banks
1998). ${ }^{5}$ Das mittels strategischer Recherche ermittelte Druckpotenzial („leverage") wird heute vielfach in Kampagnen im Rahmen von Organisierungsbemühungen eingesetzt. Weil nämlich die US-Gewerkschaften das kollektive Vertretungsrecht Betrieb für Betrieb erringen müssen und Unternehmen die gewerkschaftliche Anerkennung oft aktiv bekämpfen, geht es u.a. darum, die Unternehmen zur Neutralität zu verpflichten und insbesondere dazu, keine antigewerkschaftlichen Berater anzuheuern. Des Weiteren soll ihre Zustimmung zur Auszählung von Beitrittskarten durch einen neutralen Dritten erwirkt werden. Dieses Verfahren ist im Regelfall für die Gewerkschaften günstiger als die übliche geheime Anerkennungswahl, auch weil so die Unterstützer der Gewerkschaft im Betrieb (die Gewerkschaft selbst hat keinen $\mathrm{Zu}$ tritt) besser gegen Repressalien geschützt sind, weil sie sich nicht über einen langen Zeitraum zu erkennen geben müssen, der durch Verzögerungstaktiken des Unternehmens ausgedehnt werden kann (zur bekannten Justice for Janitors-Kampagne vgl. Dribbusch 1998; Choi in diesem Heft).

Zunächst wurde das Instrument der „strategischen Kampagne“ meist defensiv eingesetzt, zur Vermeidung oder Ergänzung von Streiks in den Abwehrkämpfen gegen unternehmerische Konzessionsforderungen. Diese defensive Ausrichtung der strategischen Kampagnen überwiegt auch heute noch bei den Gewerkschaften des verarbeitenden Gewerbes, die durch Standortverlagerungsdrohungen der Unternehmen erpressbarer sind als die Gewerkschaften des Dienstleistungssektors, wo entsprechend mehr Organizing neuer Mitglieder bzw. Betriebe stattfindet.

In der amerikanischen Debatte lassen sich verschiedene Organizing-Konzepte unterscheiden, wobei vor allem diejenigen der Dienstleistungsgewerkschaft SEIU (Service Employees International Union) und der Communication Workers of America (CWA) modellhaft sind (Moody 2007). Die SEIU setzt vorrangig auf professionell ausgebildete Organizer, oft frisch vom College, die von der Zentrale gezielt eingesetzt werden, um Betriebe zu organisieren, die in die Gesamtstrategie der Gewerkschaft passen. Anders als die meisten US-Gewerkschaften fokussiert die SEIU auf drei Kernbranchen: den öffentlichen Sektor, die Gesundheitsbranche und Immobiliendienstleistungen wie Bewachung. Kritiker dieses Konzepts konstatieren eine rein instrumentelle Mo- bilisierung der gewerkschaftlichen Basis (Moody 2007). Die CWA setzt viel stärker auf die Ausbildung der eigenen Mitgliedschaft zu Organizern und stark beteiligungsorientierte Konzepte (Early 1998). Sie brauchte allerdings wesentlich mehr Zeit als die SEIU, um ähnliche Erfolge zu erzielen. Es gibt in den USA auch eine weiterführende Diskussion um Gewerkschaften als soziale Bewegung (Social Movement Unionism), aber die Organizing-Debatte bleibt letztlich der Vorstellung von Gewerkschaften als etablierten Interessengruppen verhaftet. Insgesamt ist vor allem das SEIUKonzept einflussreich, auch weil sich diese Gewerkschaft um seine öffentlichkeitswirksame Popularisierung bemüht - die Begriffe „purple ocean“ oder „purple army“ spielen auf die gleichfarbige Präsenz von SEIUMitgliedern bei vielen politischen Veranstaltungen an (Stern 2006).

Angesichts der immer größeren Zahl von Unternehmen, die grenzüberschreitend tätig sind und verschiedene Standorte gegeneinander auszuspielen versuchen, greifen US-Gewerkschaften in ihren strategischen Kampagnen zunehmend auf transnationale Elemente zurück. Gewerkschaften beider amerikanischer Dachverbände haben ihre grenzüberschreitenden Kampagnenaktivitäten verstärkt. Gerade die Industriegewerkschaften in der American Federation of Labor and Congress of Industrial Organizations (AFL-CIO) sind allerdings oft gezwungen, eher defensiv zu agieren und mit transnationalen strategischen Druckkampagnen bestehende tarifliche Errungenschaften gegen unternehmerische Angriffe zu verteidigen, so wie z. B. derzeit die United Steelworkers in ihrer auch in Deutschland ausgetragenen Auseinandersetzung mit der Continental AG (www.solidaritaetbeiconti.de). Dagegen können die Gewerkschaften der Change to

\footnotetext{
4 Vgl. die Fallstudien zu praktischen Erfahrungen mit Strategien der US-Gewerkschaften (u. a. für Australien: Jarley et al. 2002; für Kanada: Greven 2006; für Österreich: Gstöttner-Hofer 2005; für Deutschland: Renneberg 2005).

5 Ebenfalls gebräuchlich ist der Begriff „comprehensive campaign ", meist im Zusammenhang mit Organizing-Kampagnen, bei denen neben innerbetrieblichen auch verschiedene außerbetriebliche Methoden verwendet werden, insbesondere um Druck auf das Unternehmen aufzubauen. Juravich (2007) führt den Begriff „, comprehensive strategic campaigns" ein, um das strategische Moment genauso zu betonen wie das umfassende Maßnahmenrepertoire.
} 
Win Federation (CTW), die überwiegend im Dienstleistungssektor organisieren und nicht im gleichen Maße von den Unternehmen mit Abwanderungsdrohungen erpresst werden können, die Kampagnen meist nutzen, um neue Mitglieder zu gewinnen. Sie ziehen sich dafür gelegentlich die Kritik zu, zu wenig für ihre bestehende Mitgliedschaft zu tun, während die auf Tarifverhandlungen fokussierten Gewerkschaften kritisiert werden, weil sie nicht ausreichend in Mitgliederwachstum investieren würden. Die Kritiker haben nicht ganz Unrecht, übertreiben aber in beiden Fällen.

Weil aggressive Kampagnenaktionen auch bei befreundeten Gewerkschaften, vor allem in Ländern mit sozialpartnerschaftlich organisierten industriellen Beziehungen, für Irritationen sorgen, wird in jüngerer Zeit versucht, die transnationalen Aktivitäten so zu konzipieren, dass sie für alle Beteiligten ergiebig sind. Das Global Partnerships-Programm der SEIU ist derzeit das bekannteste Projekt in diesem $\mathrm{Zu}$ sammenhang (http://www.seiu.org/about/ global_partnerships/). Organizer und Experten für strategische Recherche werden weltweit eingesetzt, um Gewerkschaften bei ihren Bemühungen zu helfen, Belegschaften zu mobilisieren und neue Mitglieder zu gewinnen, so beispielsweise in Australien, Indien, den Niederlanden, Polen, England, und auch in Deutschland (Greven 2007).

\section{1 \\ Deutsche Erfahrungen mit Organizing und strategischen Kampagnen}

In Deutschland wird die wissenschaftliche Debatte zur Krise der Gewerkschaften bisher kaum unter handlungstheoretischer Perspektive geführt. Strukturelle Erklärungen für schwindenden Einfluss dominieren, die Frage nach möglicherweise unangemessenen strategischen Reaktionen der Gewerkschaften auf sozioökonomische Veränderungen wird selten gestellt. Einen guten Überblick über die deutsche Diskussion gibt die Literaturstudie von Frerichs/Pohl (2004). Und dennoch: Trotz der großen Unterschiede zwischen den sozialpartnerschaftlichen deutschen und den konfliktorientierten US-amerikanischen industriellen Beziehungen, gibt es bereits einige Beispiele von Organizing und Kampagnen amerikanischen Stils in Deutschland.

\subsection{STRATEGIEADAPTIONEN}

Bemerkenswert sind insbesondere die Strategieadaptionen von ver.di (mit Vorläufern vor allem bei der Gewerkschaft Handel Banken Versicherungen, z. B. die erfolgreiche Kampagne zur Durchsetzung von Betriebsratswahlen bei Schlecker in den 1990er Jahren, vgl. Huhn 2001). Kampagnenstrategien amerikanischen Typs, angereichert mit Taktiken von neuen sozialen Bewegungen, wurden unter anderem gegen Xerox und Citibank (bei Letzterer sogar mit Boykottelementen) angewendet (Renneberg 2005), und seit 2004 läuft eine groß angelegte Kampagne, um bessere Arbeitsbedingungen und die Wahl von Betriebsräten bei dem Discounter Lidl durchzusetzen (vgl. den Beitrag von Matrai/ Wohland in diesem Heft). Im ver.di-Bezirk Hamburg wurde nach der Durchführung zweier Organizing-Konferenzen (www. neverworkalone.de) im Jahr 2006 sogar erstmals eine Organisierungskampagne in direkter Kooperation mit einer amerikanischen Gewerkschaft durchgeführt, wobei die SEIU insbesondere die Organizer ausbildete und anleitete (Bremme et al. 2007; Dribbusch in diesem Heft).

Da die Beteiligungsschwelle für Kampagnen- und Organizing-Aktivitäten für Gewerkschaftsmitglieder und andere Beschäftigte niedriger ist als bei Streikaktionen, wie Renneberg (2005) darlegt, ist zu erwarten, dass strategische Kampagnen und Organizing-Aktivitäten insbesondere in Branchen bzw. bei Unternehmen mit niedrigem Organisationsgrad und einem hohen Anteil prekärer Beschäftigung zukünftig stärker Verbreitung finden werden. Umgekehrt sind die öffentlichkeitsbezogenen Elemente von Kampagnen für Gewerkschaften mit hoher Präsenz in den Betrieben weniger notwendig. Aber auch die IG Metall hat in einigen Bezirken mit Strategieelementen experimentiert, die zumindest eine teilweise Orientierung an amerikanischen Vorbildern nahelegen. Hier geht es in erster Linie um ,interne Organisierung", d.h. um die stärkere Einbindung von vorhandenen Mitgliedern mit dem Ziel, Austritte zu verhindern und neue Mitglieder zu werben. In der nordrheinwestfälischen Berufsschularbeit wird unter dem Begriff „YOUnite“ ein von ver.di entwickeltes Konzept verwendet, das ebenfalls Anleihen an Organizing-Techniken macht.

\subsection{LERNPROZESSE}

Bezogen auf die oben diskutierten lerntheoretischen Einsichten und die Frage der Transnationalität ist vor allem der Lernprozess bei ver.di interessant. Bisher hat die IG Metall unter Beibehaltung der grundlegenden strategischen Ausrichtung vor allem neue Ansätze und Techniken für die Arbeit in den Betrieben erworben, ohne expliziten Bezug zu US-amerikanischen Vorbildern. Die Lernprozesse bei ver.di sind dagegen erstens eindeutig transnational angelegt; allerdings ist dies in erster Linie auf individuelles Engagement einzelner Hauptamtlicher zurückzuführen; bisher wurden mit Ausnahme der Verbindung des Bezirks Hamburg zur SEIU keine Lernstrukturen etabliert. Zweitens handelt es sich bei ver.di um Lernen vom Typ 2: die Gewerkschaft soll sich verändern, z. B. in eine „Mitmachgewerkschaft", wie es Agnes Schreieder, die die Lidl-Kampagne konzipierte, vorschwebt (Schreieder 2007). Auch erfassen die bei ver.di angestoßenen Lernprozesse die internen und externen Strategieinnovationen der amerikanischen Gewerkschaften, d.h. neben innerbetrieblichen Organisierungs-Techniken wie „mapping“ und „rating“ (s. u.) sollen auch Kapazitäten für strategische Recherche und Druckkampagnen entwickelt werden, die über das traditionelle gewerkschaftliche Instrumentarium hinausweisen. Lernen vom Typ 3 findet bisher nicht statt, aber Überlegungen zur Errichtung einer Organizing-Akademie bei ver.di legen nahe, dass in Zukunft immerhin eine Systematisierung angestrebt wird (vgl. Beitrag von Kim in diesem Heft).

Da die Anwendung amerikanischer gewerkschaftlicher Strategien in Deutschland bisher kaum als strukturierter Lernprozess organisiert ist, sondern sich eher ad hoc und experimentell auf der Basis persönlicher in den USA gesammelter Erfahrungen einzelner Hauptamtlicher oder externer Berater vollzieht, wird die US-Diskussion zu Organizing und Kampagnen bisher nur selektiv wahrgenommen, vermittelt über Berichte von deutschen Besuchern oder amerikanischen Gewerkschaftern in Deutschland. Auch in der wissenschaftlichen Auseinandersetzung wird die Komplexität der amerikanischen Diskussion bisher nur unzureichend abgebildet, und die Erfahrungen anderer Gewerkschaften mit US-Strategien, z. B. in Großbritannien, Australien, Kanada oder Österreich, wer- 
den bisher kaum verarbeitet (vgl. aber Dribbusch 2007). Wie steht es mit den Herausforderungen der institutionellen und kulturellen Passung? Welche Konzepte wurden bisher übernommen und wie wurden sie in die nationalen Gewerkschaftskulturen und Systeme der industriellen Beziehungen eingepasst?

\subsection{AUSGANGSBEDINGUNGEN - ÄHNLICHKEITEN UND UNTERSCHIEDE}

Bemerkenswert sind zunächst die Ähnlichkeiten in Bezug auf das Klima der Angst, das gewerkschaftliche Organisierungsbemühungen heute wieder bestimmt. Wie ihre amerikanischen Kollegen müssen deutsche Gewerkschafter bzw. Aktivisten vor allem im Dienstleistungssektor mit Einschüchterungen und Repressalien rechnen. Systembedingt unterschiedlich ist nur, dass sich diese vor allem gegen die Einrichtung von Betriebsräten richten, die anti-gewerkschaftlichen Techniken gleichen sich. Während die Abwanderungsdrohung im verarbeitenden Gewerbe in den USA zurzeit noch glaubwürdiger ist als in Deutschland, gleicht sich aber der allgemeine Kontext ständiger Wettbewerbsverschärfung und damit verbundener Forderungen an die Beschäftigten und Gewerkschaften.

Einige der zentralen Techniken des Organizing, insbesondere das „mapping“ des Betriebs, d.h. der genauen Bestimmung der einzelnen Arbeitsplätze im Betrieb und die direkte Ansprache der Beschäftigten, auch privat, lassen sich relativ konfliktfrei transferieren, auch weil sie an etablierte oder frühere Praktiken anknüpfen. Allerdings stellt auch die gezielte Ansprache von Beschäftigten für haupt- wie ehrenamtliche Gewerkschafter durchaus eine Herausforderung dar (Dribbusch 2003, S. 275).

Ungewohnt ist dagegen das ,rating", die Bewertung von Beschäftigten in Bezug auf ihre Einstellung zu Gewerkschaften. Noch schwieriger ist der Rückgriff auf das Konzept des „Leader“. Führungspersönlichkeiten nehmen in den amerikanischen Konzepten eine zentrale Funktion ein, um die für Mapping und Rating notwendigen $\mathrm{Da}$ ten zu ermitteln und dann die Überzeugungsarbeit für die Gewerkschaft zu leisten. Diese sprachlich-kulturellen Empfindlichkeiten werden gewöhnlich mit dem Begriff „Schlüsselpersonen“ umschifft.

Während aber die einzelnen Techniken recht problemlos übertragbar scheinen, bestehen größere Schwierigkeiten bei der grundsätzlichen strategischen Ausrichtung der amerikanischen Organizing-Strategien. Alle Aktivitäten und Konzepte sind nämlich auf das zentrale Datum des Erringens des gewerkschaftlichen Kollektivvertretungsanspruchs ausgerichtet, das es im deutschen System freiwilliger individueller Mitgliedschaft und überwiegend zentralisierter Tarifverhandlungen nicht gibt. Am ehesten könnte die Betriebsratswahl als deutsches Äquivalent fungieren, aber gerade die Existenz von Betriebsräten in ihrer institutionellen und kulturellen Komplexität kommt ja in den amerikanischen Vorbildkonzepten nicht vor. Und so werden die Organizing-Techniken bisher auch nicht primär für die Wahl gewerkschaftlich starker Betriebsräte eingesetzt (am ehesten noch bei der Lidl-Kampagne und bei der für diese vorbildhaften Schlecker-Kampagne). Dass die Mitgliedergewinnung nicht im Mittelpunkt der Kampagnen steht, ist nach unserer Einschätzung eine sinnvolle Anpassung der amerikanischen Konzepte, da in Deutschland Mitgliederwerbung über individuelle Ansprache im Betrieb durch Betriebsräte, Vertrauensleute oder aktive Mitglieder - jederzeit erfolgen kann und nicht zu einem bestimmten Zeitpunkt kollektiv vonstattengehen muss. Im Vordergrund stehen eher die Durchsetzung von bestehenden oder neuen Tarifverträgen sowie die Verbesserung des gewerkschaftlichen Images bei den Beschäftigten wobei all dies bei der individuellen Ansprache bezüglich einer Mitgliedschaft hilft. Und dennoch: Zur kulturellen Passung der amerikanischen Organizing-Strategien als Strategien zur Mitgliedergewinnung fehlt die Einarbeitung der komplexen Beziehung Gewerkschaft-Betriebsrat und die Entwicklung politischer Mobilisierungskonzepte für das System freiwilliger Mitgliedschaft.

Strategische Kampagnen sind in Deutschland bisher selten versucht worden, und auch die Intensität ihrer Vorbereitung, d.h. die gezielte strategische Analyse des unternehmerischen Umfelds zur Identifizierung von Druckpunkten („leverage“), war bislang nur gering. Die Informationsbeschaffung in Deutschland wird oft aufgrund geringer ausgeprägter Informationspflichten und -rechte als wesentlich problematischer eingeschätzt als in den USA. Der geringeren allgemeinen Transparenz stehen hierzulande jedoch legale Formen der Mitbestimmung in Betriebsräten und Aussichtsräten bzw. indus- trieweite Tarifverhandlungen gegenüber, die diesen Mangel unter Umständen vermindern oder gar aufheben könnten. Allerdings bedürfte es zur Nutzung dieser spezifischen deutschen Informationsquellen der Bereitschaft, „schlechte“ Arbeitgeber auch wirtschaftlich zu schädigen oder gar vom Markt zu verdrängen. Bisher gibt es hier große Berührungsängste, die aufgrund etablierter sozialpartnerschaftlicher Beziehungen auch verständlich sind. Strategische Recherche wird daher bisher eher ad hoc und zögerlich eingesetzt. Mit der Schwächung des traditionellen Kampfrepertoires und der zunehmenden Konfrontation mit den Kampagnen von USGewerkschaften, die deutsche Unternehmen unter Druck setzen (vgl. das Beispiel Continental AG oben), könnte aber auch in Deutschland eine systematische Diskussion strategischer Kampagnenfähigkeit notwendig und sinnvoll sein. Erste Anzeichen dafür gibt es durchaus.

\section{Jenseits der kontrollierten Mobilisierung}

Die von deutschen Gewerkschaften begonnenen Lernprozesse sind für ihre strategische Erneuerung unerlässlich. Insbesondere der Blick auf die Erfahrungen in anderen Ländern erlaubt es, die konzeptionellen Grenzen der eigenen institutionellen Einbindung zu durchbrechen und das strategische Handlungsrepertoire zu erweitern. Dabei ist wichtig, die Strategieadaption systematisch zu betreiben und nicht durch die unbedachte Übernahme von Teilaspekten etablierte Institutionen zu beschädigen. Ein Blick auf die Erfahrungen, die andere mit transnationalen Lernprozessen machen, könnte hier helfen. In Großbritannien wie auch in den USA selbst hat der verengte Blick auf Organizing einige Kritik erfahren, weil die teilweise zentralisierte und top-down ausgerichtete Kampagnenführung und die "kontrollierte Mobilisierung “ (Heery et al. 2000) für wenig nachhaltig gehalten werden (Fairbrother 2004). Ohne tiefer gehende Veränderungen der gewerkschaftlichen Strukturen, die auf eine demokratische, beteiligungsorientierte Gewerkschaftskultur zielen, drohten „Organisierungsstrohfeuer“, die nicht von Dauer sind. 
Argyris, C./Schön, D. A. (1999): Die Lernende Organisation. Grundlagen, Methode, Praxis, Stuttgart

Bandelow, N. C. (2003): Policy Lernen und politische Veränderungen, in: Schubert, K./Bandelow, N. C. (Hrsg.): Lehrbuch der Politikfeldanalyse, München und Wien, S. 289-331

Banks, A. (1998): Strategische Gewerkschaftskampagnen in den Vereinigten Staaten von Amerika, in: Gstöttner-Hofer, G./Greif, W./Kaiser, E./ Deutschbauer P. (Hrsg.): Mobilisierung und Kampagnenfähigkeit. Impulse für die gewerkschaftliche Interessendurchsetzung, Wien, S. 51-65

Banks, A./Metzgar, J. (1993): Von der Stellvertreterpolitik zur sozialen Bewegung, in: Jahrbuch Arbeit und Technik 1993, Bonn, S. 291-299 Behrens, M. (2005a): Mitgliederrekrutierung und institutionelle Grundlagen der Gewerkschaften. Deutschland im internationalen Vergleich, in: Berliner Debatte Initial 5, S. 30-37

Behrens, M. (2005b): Die Rolle der Betriebsräte bei der Werbung von Gewerkschaftsmitgliedern, in: WSI-Mitteilungen 6, S. 329-338

Bremme, P./Fürniß, U./Meinecke, U. (Hrsg.) (2007): Never work alone. Organizing - ein Zukunftsmodell für Gewerkschaften, Hamburg Bronfenbrenner, K./Juravich, T./Friedman, S./Hurd, R./Oswald, R./Seeber, R. (Hrsg.) (1998): Organizing to Win. New Research on Union Strategies, Ithaca, NY

Dribbusch, H. (1998): Mitgliedergewinnung durch offensive Interessenvertretung. Neue gewerkschaftliche Organisierungsansätze aus den USA - Das Beispiel "Justice for Janitors“, in: WSI-Mitteilungen 5, S. 281-291 Dribbusch, H. (2003): Gewerkschaftliche Mitgliedergewinnung im Dienstleistungssektor. Ein Drei-Länder-Vergleich im Einzelhandel, Berlin Dribbusch, H. (2007): Das „Organizing-Modell“ . Entwicklung, Varianten und Umsetzung, in: Bremme, P./Fürniß, U./Meinecke, U. (Hrsg.) (2007): Never work alone. Organizing - ein Zukunftsmodell für Gewerkschaften, Hamburg, S. 24-52

Early, S. (1998): Membership-Based Organizing, in: Mantsios, G. (Hrsg.): A New Labor Movement for the New Century, New York, S. 82-103 Fairbrother, P. (2004): Rediscovering Union Democracy: Process of Union Revitalisation and Renewal, in: Rediscovering union democracy: Processes of union revitalization and renewal. Labor History 3, S. 368-376

Fairbrother, P. D./Yates, C. (Hrsg.) (2003): Trade Unions in Renewal, A Comparative Study, London/New York

Frege, C. (1999): Union Organizing in a Comparative Perspective. The Case of Germany, Vortrag bei der Jahrestagung der American Political Science Association, Atlanta, Georgia, 2.-5. September

Frege, C. (2000): Gewerkschaftsreformen in den USA. Eine kritische Analyse des ,Organisierungsmodells', in: Industrielle Beziehungen 3, S. 260280

Frege, C./Kelly, J. (2003): Union Revitalization Strategies in Comparative Perspective, in: European Journal of Industrial Relations 1, S. 7-24

Frerichs, P./Pohl, W. (2004): Zukunft der Gewerkschaften, Düsseldorf: Hans-Böckler-Stiftung, Arbeitspapier 44, Teil 1

Greven, T. (2003): Clash of Globalizations? The Politics of International Labor Rights in the United States, Frankfurt

Greven, T. (2006): Learning from the U. S.? Canadian Unions and the Cross-Border Diffusion of Labour Movement Strategies, in: Zeitschrift für Kanadastudien 2, S. 118-136

Greven, T. (2007): Die SEIU spielt die transnationale Karte, in: Mitbestimmung 12 , S. 26-29
Greven, T./Scherrer, Ch. (2005): Globalisierung gestalten. Weltökonomie und Sozialstandards, Bonn

Gstöttner-Hofer, G. (2005): Kampagnen als Erweiterung des gewerkschaftlichen Strategierepertoires in Österreich. Ursachen - Erfahrungen Erkenntnisse, Wien

Heery, E/Simms, M./Delbridge, R./Salmon, J./Simpson, D. (2000): Union Organizing in Britain: A Survey of Theory and Practice, in: International Journal of Human Resources Management 5, S. 986-1007 Huhn, J. (2001): Die Schlecker-Kampagne 1994-1995, Mannheim/Heidelberger HBV-Hefte: Ideen und Aktionen an der Basis, hrsg. von ver.di (ehem. HBV-BZV Mannheim-Heidelberg)

Hurd, R./Milkman, R./Turner, L. (2003): Reviving the American Labour Movement: Institutions and Moblization, in: European Journal of Industrial Relations 1, S. 99-117

Hyman, R. (2007): How can trade unions act strategically?, in: Transfer 2, S. $193-210$

Jarley, P./Harley, B./Hall, R. (2002): Innovation in Australians Trade Unions, in: Industrial Relations 2, S. 228-248

Juravich, T. (2007): Beating Global Capital: A Framework and Method for Union Strategic Corporate Research and Campaigns, in: Bronfenbrenner, K. (Hrsg.): Global Unions. Challenging Transnational Capital Through Cross-Border Campaigns, Ithaca, NY, S. 16-39.

Kochan, Th./Katz, H./McKersie, R. (1986): The Transformation of American Industrial Relations, New York.

Milkman, R./Voss, K. (2004): Rebuilding Labor. Organizing and organizers in the New Union Movement, Ithaca, NY

Moody, K. (2007): U. S. Labor in Trouble and Transition: The Failure of Reform from Above, the Promise of Revival from Below, London Renneberg, P. (2005): Die Arbeitskämpfe von morgen? Arbeitsbedingungen und Konflikte im Dienstleistungsbereich, Hamburg

Rose, R. (1991): What is Lesson-Drawing?, in: Journal of Public Policy 1 , S. 3-30

Sabatier, P. A. (1993): Policy Change over a Decade or More, in: Sabatier, P. A./Jenkins-Smith, H. C. (Hrsg.): Policy change and learning: An advocacy coalition approach, Boulder, S. 13-39

Scherrer, Ch. (2005): Can Germany Learn from the USA?, in: Stefan B./Klobes, F./Scherrer, Ch. (Hrsg.): Surviving Globalization? Perspectives for the German Economic Model, Dordrecht, S. 15-31

Schreieder, A. (2007): Die Lidl-Kampagne - ein Zukunftsmodell für Gewerkschaften, in: Bremme, P./Fürniß, U./Meinecke, U. (Hrsg.) (2007):

Never work alone. Organizing - ein Zukunftsmodell für Gewerkschaften, Hamburg, S. 153-174

Stern, A. (2006): A Country That Works. Getting America Back on Track, New York

Tattersall, A. (2007): Labor-Community Coalitions, Global Union Alliances and the Potential of SEIU's Global Partnerships, in: Bronfenbrenner, K. (Hrsg.): Global Unions. Challenging Transnational Capital Through CrossBorder Campaigns, Ithaca, S. 155-173

Turner, L./Katz, H./Hurd, R. (Hrsg.) (2001): Rekindling the Movement. Labor's Quest for Relevance in the 21 ${ }^{\text {st }}$ Century, Ithaca

Visser, J. (1994): European Trade Unions. The Transition Year, in: Hyman, R./Ferner, A. (Hrsg.): New Frontiers in Industrial Relations, Oxford, Cambridge/MA, S. 80-107

Zoll, R. (2003): Gewerkschaften als lernende Organisationen - was ist eigentlich das Problem?, in: Gewerkschaftliche Monatshefte 5, S. 315-321 